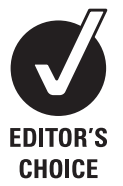

CHOICE

\section{See Editorial, p 431}

'Department of Vascular Medicine, Academic Medical Centre, Amsterdam, The Netherlands

${ }^{2}$ Department of Clinical Epidemiology, Biostatistics and Bioinformatics, Academic Medical Centre, Amsterdam, The Netherlands

Correspondence to Dr Sara-Joan Pinto-Sietsma, Department of Clinical Epidemiology, Biostatistics and Bioinformatics, Academic Medical Centre, Postbus 22660, Amsterdam 1100 DD, The Netherlands; s.j.pinto@amc.nl

Accepted 18 November 2011 Published Online First 22 December 2011

\title{
Arterial stiffness is increased in families with premature coronary artery disease
}

\author{
Ties A Mulders, ${ }^{1}$ Bas van den Bogaard, ${ }^{1}$ Annemieke Bakker, ${ }^{1}$ Mieke D Trip, ${ }^{1}$ \\ Erik S Stroes, ${ }^{1}$ Bert-Jan H van den Born, ${ }^{1}$ Sara-Joan Pinto-Sietsma ${ }^{1,2}$
}

\begin{abstract}
Objective A positive family history of premature coronary artery disease (CAD) is a risk factor for cardiovascular disease (CVD), independent of traditional risk factors. Therefore, currently used risk algorithms poorly predict risk in these individuals. Novel methods are thus needed to assess cardiovascular risk. Pulse-wave velocity (PWV) might be such a method, but it is unknown whether PWV is increased in first-degree relatives of patients with premature CAD.

Design Observational case-control study.

Setting Academic hospital.

Patients Patients with premature CAD and a positive family history of premature CVD $(n=50)$, their firstdegree relatives without CVD $(n=50)$ and unrelated controls $(n=50)$.
\end{abstract}

\section{Interventions None.}

Main Outcome Measures PWV was measured with using an Arteriograph system. Differences in PWV were assessed by a generalised linear model and multinomial logistic regression.

Results Patients with premature CAD had a higher PWV compared with first-degree relatives and controls $(9.69 \pm 2.90 \mathrm{~m} / \mathrm{s}$ vs $8.15 \pm 1.96 \mathrm{~m} / \mathrm{s}$ and $7.38 \pm 1.08 \mathrm{~m} /$ $\mathrm{s} ; \mathrm{p}<0.05$ patients vs all groups). Linear regression showed all groups related to PWV, with patients having the highest PWV and controls the lowest $(p<0.0001)$. Furthermore, PWV was associated with first-degree relatives (OR 1.32, 95\% $\mathrm{Cl} 1.02$ to $1.72 ; p<0.05)$ and premature CAD (OR 1.72, 95\% Cl 1.32 to 2.24; $p<0.05$ ) compared with controls. These findings were independent of blood pressure and other traditional risk factors.

Conclusions Patients with premature CAD and their first-degree relatives had higher PWV compared with controls, independent of other risk factors. This holds promise for the future, in which arterial stiffness might play a role in risk prediction within families with premature CAD.

A positive family history of premature coronary artery disease (CAD) is an important risk factor for cardiovascular disease (CVD) and is, in fact, independent of other risk factors. ${ }^{1-3}$ The associated risk increases further when relatives are affected at a younger age, with an OR of 1.3 in individuals with relatives affected below 55 years, to OR of 10 and higher in individuals with relatives affected below 45 years of age. ${ }^{4}$

Whereas a positive family history of CVD identifies whole families at risk, it fails to identify which specific kindred are at risk within the family.
This emphasises the need to refine risk further among siblings in these families. Traditional risk score algorithms poorly predict cardiovascular risk in general, but even more in relatives of patients with premature $\mathrm{CAD} .^{6}$ The latter reflects the fact that these individuals are referred for cardiovascular risk evaluation at a relatively young age, whereas in the traditional risk score algorithms age is the most potent factor determining risk. In addition, risk score algorithms use markers of risk in stead of identifying disease itself. Therefore, investigators keep searching for practical tools to assess subclinical disease, to identify individuals with early-onset CVD.

Pulse-wave velocity (PWV), the gold standard of arterial stiffness, ${ }^{7}$ has emerged as a novel biomarker for predicting cardiovascular mortality and morbidity, independent of traditional cardiovascular risk factors. ${ }^{8}$ Overall, arterial stiffness only poorly predicts cardiovascular risk, which may relate to the heterogeneity of the studied populations. Interestingly, a recent prospective trial concluded that PWV measurement could be particularly useful in younger individuals with a genetic predisposition to CVD. ${ }^{9}$ To date, this hypothesis has not been tested. In the present study, we therefore evaluated whether 'healthy' first-degree relatives without overt CVD of patients with premature $\mathrm{CAD}$ are characterised by an increased PWV compared with controls with a negative family history of CVD. To test this, we assessed PWV in patients with premature $\mathrm{CAD}$, their first-degree relatives and unrelated controls.

\section{METHODS \\ Study design}

We recruited 50 patients with premature CAD and a positive family history of premature CVD. We were thus able to select a population of individuals with a genetic predisposition to CVD. In the families of these participants, we also recruited 50 first-degree relatives without overt CVD. In addition, we recruited 50 unrelated controls without overt CVD. We matched for gender and age.

The primary objective of the study was to evaluate whether there are differences in PWV between patients with premature $\mathrm{CAD}$, their first-degree relatives and controls. The secondary objective was to explore the association between PWV and patients with premature CAD and their first-degree relatives with and without adjustment of possible confounders. 
In all groups we assessed traditional risk factors for CVD according to the standard procedures in our hospital. For the first-degree relatives and controls, we assessed the Framingham risk score.

The first-degree relatives and controls were not allowed to have a history of CVD and were excluded if they had any symptoms of CVD, both of which were assessed by a standardised questionnaire. Furthermore, controls were not allowed to use any medication or to have a positive family history of CVD. Furthermore, we assessed coronary artery calcification (CAC) scores in the first-degree relatives, to evaluate subclinical atherosclerosis.

To minimise further the influence of other cardiovascular risk factors, individuals in all groups were excluded if they had severe hypertension (above 180/110 mm Hg), diabetes mellitus or known familial hypercholesterolaemia. Individuals were excluded if they were under the age of 18 years, if they were unable to give informed consent or in the case of pregnancy or lactation.

This work was conducted in accordance with the Declaration of Helsinki. All participants gave written informed consent and the study was approved by the local Institutional Review Board.

\section{Definitions}

$\mathrm{CAD}$ was defined as an acute myocardial infarction or $\mathrm{CAD}$, needing revascularisation by percutaneous coronary intervention or coronary artery bypass grafting. Premature CAD is usually defined as an event occurring before the age of 55 years in men and 60 years in women. ${ }^{10}$ To increase the likelihood of including families with a genetic predisposition to CVD, the age limits were lowered to 41 years in men and 46 years in women. A positive family history was defined as one or more first-degree and/or two or more second-degree family members with CVD before the age of 51 years in men and 56 years in women, in line with the GENECARD definition, found in the literature. ${ }^{10} \mathrm{CVD}$ was defined in the same manner as $\mathrm{CAD}$, with the extension of strokes and peripheral artery disease necessitating percutaneous transluminal angioplasty or bypass surgery.

Hypertension and hypercholesterolaemia were defined in patients as the use of blood pressure or cholesterol-lowering medication before the first event or, in the case of a first-degree relative, as medication use at the time of the study visit. Blood pressure-lowering medication use was defined as the use of betablockers, calcium antagonists, ACE inhibitors, angiotensin II receptor antagonists or diuretics. In individuals who did not use medication, hypertension was defined as a blood pressure above $140 / 90 \mathrm{~mm} \mathrm{Hg}$ in rest and hypercholesterolaemia as fasting total cholesterol levels above $6.2 \mathrm{mmol} / \mathrm{l}$, as defined by the third report of the National Cholesterol Education Program. ${ }^{11}$ Smoking was defined as current smoking or past smoking 5 years ago or less.

\section{Pulse-wave velocity}

Participants visited the hospital after an overnight fast and were asked to refrain from smoking at least $8 \mathrm{~h}$ before the visit. All measurements were performed in a supine position after $15 \mathrm{~min}$ rest in a quiet, temperature-controlled room. Arterial stiffness was assessed using the Arteriograph system (Tensiomed Kft., Budapest, Hungary), which shows close correlation with the widely used Sphygmocor system. ${ }^{12}$ The Arteriograph is an operator-independent non-invasive device, which uses oscillometric pressure curves registered by an upper arm blood pressure cuff to determine blood pressure and PWV as validated, ${ }^{13}$ described and illustrated previously. ${ }^{14}$ In short, PWV measurements are performed when cuff pressure exceeds systolic blood pressure by $35-40 \mathrm{~mm} \mathrm{Hg}$, with a completely occluded brachial artery. The measurement is based on the fact that during systole, blood volume ejected into the aorta generates a pulse wave, the so-called 'early systolic peak'. As this pulse wave runs down the periphery, it reflects from the bifurcation of the aorta, creating a second wave, the 'late systolic peak'. The return time of the pulse waves was calculated as the time difference between the first and the reflected systolic wave. PWV is calculated from this transit time and the distance travelled by the pulse wave. Estimation of the distance travelled by the pulse wave (from the heart to the bifurcation and back), is based on measuring the distance between the sternal notch and the pubic symphysis using a tape measure. PWV was recorded as continuous data. Also, the percentage of individuals with a PWV above $12 \mathrm{~m} / \mathrm{s}$ was assessed among the groups, which is comparable with subclinical organ damage according to the 2007 European Society of Hypertension guidelines. ${ }^{15}$

\section{Coronary artery calcification}

In all first-degree relatives we performed a coronary CT scan to assess the presence of coronary lesions through CAC. All CT scans were performed using a 64-slice multidetector CT scanner (Philips Medical Systems, Best, The Netherlands). The scanning protocol was as follows: tube voltage, $120 \mathrm{kV}$; tube current, $55 \mathrm{mAs}$; detector collimation, $40 \times 2.5 \mathrm{~mm}$; gantry rotation, $420 \mathrm{~ms}$. The data were transferred to a post-processing workstation (Extended Brilliance Workplace; Philips Medical Systems). We recorded CAC for the main arteries; the total score was calculated by summing lesion scores of all sections. We evaluated CAC according to Agatston et al ${ }^{16}$ and expressed further as age/sex percentiles. In a secondary analysis, firstdegree relatives were divided into two groups, according to the results of the CAC. A score above the 80th percentile was considered to be abnormal, consistent with the literature, ${ }^{17}$ lower scores were considered to be normal.

\section{Statistical analyses}

Sample size was calculated by a pilot study, in which we found a difference in PWV between patients and controls of $1.30 \pm 2.1 \mathrm{~m} / \mathrm{s}$. Using a power of 0.80 and a $\mathrm{p}$ value of 0.05 , a calculated sample size of 48 individuals was needed in each group.

Differences in baseline characteristics were assessed between the three groups by using $\chi^{2}$ tests (in the case of proportions), or analysis of variance (in the case of continuous data) and individual group comparison was done by Fisher's least significant difference correction.

The association of PWV as a continuous variable with the different groups was assessed in two different ways. First, we computed OR using multinomial logistic regression, considering the PWV, as continuous variable and the different groups as outcome. In this analysis, all first-degree relatives were used as one group. We adjusted the crude model (model 1) for age and sex (model 2) and, finally, for other confounders such as hypercholesterolaemia, smoking and systolic blood pressure (model 3). We corrected for systolic blood pressure, as PWV is directly dependent on the blood pressure during the measurement. ${ }^{18}$ Furthermore, we corrected for factors that differed significantly among the groups. In this model, we used the controls as a reference category. Second, we performed a generalised linear model, which considers the different groups as variable and the PWV as outcome measure.

To test whether there were differences in PWV in first-degree relatives according to the CAC score, we performed 
a multinomial logistic regression in the same manner as described above, but now dividing the first-degree relatives into two groups: one with CAC scores above the 80th percentile and one with CAC scores below this point.

Finally, to assess influences of family relations, we analysed our data in a mixed model, in which family relation was included as a paired variable. This had no influence on the outcome and the results remained similar (data not shown).

A p value less than 0.05 was considered statistically significant. Data were analysed using SPSS software V.16.0.

\section{RESULTS}

\section{Baseline characteristics}

The characteristics of the participants are listed in table 1 . The groups were well matched for both age and sex. We found that first-degree relatives were more often smokers (38\% vs $20 \%$; $\mathrm{p}<0.05)$, had higher total cholesterol levels $(5.4 \pm 0.9 \mathrm{mmol} / \mathrm{l}$ vs $5.0 \pm 0.8 \mathrm{mmol} / \mathrm{l} ; \mathrm{p}<0.05)$ and higher low-density lipoprotein cholesterol levels $(3.5 \pm 0.9 \mathrm{mmol} / \mathrm{l}$ vs $3.1 \pm 0.7 \mathrm{mmol} / \mathrm{l} ; \mathrm{p}<0.05)$ compared with controls. The Framingham risk score was somewhat higher in first-degree relatives compared with controls, but did not reached statistical significance. Patients with premature CAD had higher glucose levels $(5.5 \pm 0.8 \mathrm{mmol} / \mathrm{l}$ vs $5.2 \pm 0.7 \mathrm{mmol} / 1$ and $5.1 \pm 0.4 \mathrm{mmol} / 1 ; \mathrm{p}<0.05)$, lower total cholesterol levels $(4.2 \pm 1.0 \mathrm{mmol} / \mathrm{l}$ vs $5.4 \pm 0.9 \mathrm{mmol} / \mathrm{l}$ and $5.0 \pm 0.8 \mathrm{mmol} / \mathrm{l} ; \quad \mathrm{p}<0.05)$, lower low-density lipoprotein cholesterol levels $(2.2 \pm 0.7 \mathrm{mmol} / \mathrm{l}$ vs $3.5 \pm 0.9 \mathrm{mmol} / \mathrm{l}$ and $3.1 \pm 0.7 \mathrm{mmol} / \mathrm{l} ; \quad \mathrm{p}<0.05)$ and higher triglycerides $(2.1 \pm 3.8 \mathrm{mmol} / \mathrm{l}$ vs $1.1 \pm 0.6 \mathrm{mmol} / \mathrm{l}$ and $1.0 \pm 0.5 \mathrm{mmol} / \mathrm{l}$; $\mathrm{p}<0.05)$ compared with first-degree relatives and controls. The patients also more often used antihypertensive and cholesterollowering medication, mostly for secondary prevention reasons.

With regard to blood pressure, systolic blood pressure was comparable between the groups (patients $130.7 \pm 18.5 \mathrm{~mm}$

Table 1 Baseline characteristics of the participants

\begin{tabular}{llll}
\hline & $\begin{array}{l}\text { Controls } \\
(\mathbf{n}=\mathbf{5 0})\end{array}$ & $\begin{array}{l}\text { First-degree } \\
\text { relatives }(\mathbf{n}=\mathbf{5 0})\end{array}$ & $\begin{array}{l}\text { Patients with } \\
\text { CAD (n=50) }\end{array}$ \\
\hline Age, years & $45.6 \pm 6.6$ & $45.6 \pm 7.9$ & $46.0 \pm 3.6$ \\
Male gender, n (\%) & $27(54)$ & $27(54)$ & $27(54)$ \\
Smoking, n (\%) & $10(20)$ & $19(38)^{*}$ & $28(56)^{*}$ \\
SBP, mm Hg & $125.2 \pm 15.6$ & $129.7 \pm 11.3$ & $130.7 \pm 18.5$ \\
DBP, mm Hg & $79.4 \pm 9.9$ & $82.6 \pm 8.8$ & $84.8 \pm 10.4^{*}$ \\
BMl, kg/m² & $23.0 \pm 4.4$ & $23.7 \pm 3.9$ & $24.4 \pm 5.0$ \\
Glucose, mmol/l & $5.1 \pm 0.4$ & $5.2 \pm 0.7$ & $5.5 \pm 0.8^{*} \dagger$ \\
Total cholesterol, mmol/l & $5.0 \pm 0.8$ & $5.4 \pm 0.9^{*}$ & $4.2 \pm 1.0^{*} \dagger$ \\
HDL-cholesterol, mmol/l & $1.5 \pm 0.4$ & $1.4 \pm 0.4$ & $1.2 \pm 0.3^{*} \dagger$ \\
LDL-cholesterol, mmol/l & $3.1 \pm 0.7$ & $3.5 \pm 0.9^{*}$ & $2.2 \pm 0.7^{*} \dagger$ \\
Triglycerides, mmol/l & $1.0 \pm 0.5$ & $1.1 \pm 0.6$ & $2.1 \pm 3.8^{*} \dagger$ \\
Creatinine, mmol/l & $72.9 \pm 11.2$ & $72.5 \pm 11.9$ & $70.7 \pm 13.6$ \\
Hypertension, $\mathrm{n}(\%)$ & $0(0)$ & $6(12)$ & $10(20)^{*}$ \\
Hypercholesterolaemia, $\mathrm{n}(\%)$ & $0(0)$ & $7(14)^{*}$ & $11(22)^{*} \dagger$ \\
Framingham risk score & $2.3(1.2 ; 3.3)$ & $3.2(1.2 ; 4.8)$ & - \\
Medication use & & & \\
$\quad$ Antihypertensive, $\mathrm{n}(\%)$ & $0(0)$ & $5(10)$ & $46(92)^{*} \dagger$ \\
$\quad$ Cholesterol lowering, $\mathrm{n}(\%)$ & $0(0)$ & $6(12)$ & $46(92)^{*} \dagger$ \\
PWV, m/s & $7.5 \pm 1.2$ & $8.2 \pm 1.9$ & $9.6 \pm 2.9^{*} \dagger$ \\
PWV >12 m/s, n (\%) & $0(0)$ & $3(6)$ & $10(20)^{*} \dagger$ \\
\hline
\end{tabular}

Continuous data are expressed as mean \pm SD except for the Framingham risk score, which is expressed as median (25th; 75th percentiles), categorical data as absolute numbers with (percentages).

${ }^{*} p<0.05$ compared with controls

$\dagger p<0.05$ compared with first-degree relatives.

$B M I$, body mass index; $C A D$, coronary artery disease; $D B P$, diastolic blood pressure; $H D L$, high-density lipoprotein; LDL, low-density lipoprotein; PWV, pulse-wave velocity; SBP, systolic blood pressure.

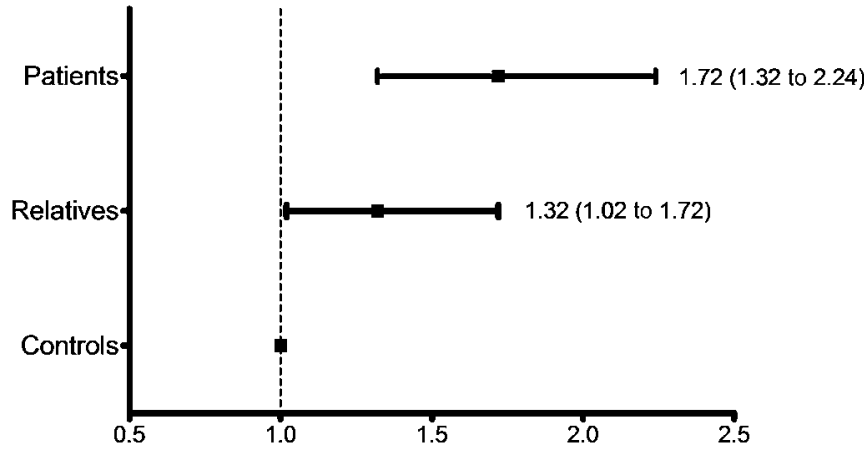

Figure 1 Association of pulse-wave velocity (PWV) with the different groups, assessed by multinomial logistic regression (OR, 95\% CI). The reference for the calculated $\mathrm{OR}$ consisted of the controls.

$\mathrm{Hg}$; first-degree relatives $129.7 \pm 11.3 \mathrm{~mm} \mathrm{Hg}$; controls $125.2 \pm 15.6 \mathrm{~mm} \mathrm{Hg}$ ). Diastolic blood pressure was higher in patients compared with controls $(84.8 \pm 10.4 \mathrm{~mm} \mathrm{Hg}$ vs 79.4 \pm 9.9 mm Hg; $<$ 0.05).

Unadjusted PWV of first-degree relatives did not significantly differ from controls $(8.2 \pm 1.9 \mathrm{~m} / \mathrm{s}$ vs $7.5 \pm 1.2 \mathrm{~m} / \mathrm{s})$, whereas patients had higher PWV compared with first-degree relatives and controls $(9.6 \pm 2.9 \mathrm{~m} / \mathrm{s}$ vs $8.2 \pm 1.9 \mathrm{~m} / \mathrm{s}$ and $7.5 \pm 1.2 \mathrm{~m} / \mathrm{s}$; $\mathrm{p}<0.05)$.

\section{Association between PWV and the different groups}

In the multinomial logistic regression, PWV was positively associated with both patients (OR $1.72,95 \%$ CI 1.32 to 2.24 ; $\mathrm{p}<0.05)$ and first-degree relatives (OR 1.32, 95\% CI 1.02 to 1.72 ; $\mathrm{p}<0.05$ ) compared with controls (figure 1 and table 2). This association retained statistical significance after adjustment for confounders (models 2 and 3). PWV was also positively associated with patients compared with all first-degree relatives (OR 1.30, 95\% CI 1.08 to 1.57 ; $<<0.05$ ). Again, this association remained after adjustment for confounders. These findings were confirmed in the linear regression analysis, which showed all three groups to be linearly related to PWV $(p<0.0001$, data not shown).

\section{Association between PWV and different groups according to CAC results}

Assuming a hereditary component in the families, the firstdegree relatives might consist of individuals who have and who have not inherited the genetic defect. As we do not know the specific defect in the families, we chose to evaluate subclinical atherosclerosis by means of CAC. After dividing the first-degree relatives into a group with a high and a group with a normal CAC score, we found that $34 \%(n=17)$ of all first-degree relatives

Table 2 Multinomial logistic regression to assess the relation between PWV and patients, first-degree relatives and controls

\begin{tabular}{llll}
\hline Group & Controls & First-degree relatives & Patients with CAD \\
\hline PWV & & & \\
Model 1 & 1.00 & $1.32(1.02-1.72)^{*}$ & $1.72(1.32-2.24)^{*} \dagger$ \\
Model 2 & 1.00 & $1.38(1.04-1.83)^{*}$ & $1.91(1.43-2.57)^{*} \dagger$ \\
Model 3 & 1.00 & $1.51(1.05-2.17)^{*}$ & $2.18(1.49-3.19)^{*} \dagger$ \\
\hline
\end{tabular}

Model 1, crude model; model 2, adjusted for age and sex; model 3, additionally adjusted for hypercholesterolaemia, smoking and systolic blood pressure. Controls are used as reference.

${ }^{*} \mathrm{p}<0.05$ compared with controls.

$+p<0.05$ compared with first-degree relatives

$C A D$, coronary artery disease; PWV, pulse-wave velocity. 
Table 3 Multinomial logistic regression to assess the relation between PWV and patients, first-degree relatives with and without high CAC score and controls

\begin{tabular}{|c|c|c|c|c|}
\hline \multirow[b]{2}{*}{ Group } & \multirow[b]{2}{*}{ Controls } & \multicolumn{2}{|c|}{ First-degree relatives } & \multirow[b]{2}{*}{ Patients } \\
\hline & & Normal CAC & Abnormal CAC & \\
\hline \multicolumn{5}{|l|}{ PWV } \\
\hline Model 1 & 1.00 & $1.23(0.91-1.65)$ & $1.49(1.09-2.04)^{*}$ & $1.72(1.32-2.24)^{*} \dagger$ \\
\hline Model 2 & 1.00 & $1.31(0.96-1.79)$ & $1.53(1.08-2.17)^{*}$ & $1.91(1.43-2.57)^{*} \dagger$ \\
\hline Model 3 & 1.00 & $1.46(0.99-2.14)$ & $1.61(1.02-2.54)^{*}$ & $2.18(1.49-3.19)^{*} \dagger$ \\
\hline
\end{tabular}

had a high CAC score (above the 80th percentile). In the multinomial logistic regression, we found that PWV was positively associated with both patients (OR 1.72, 95\% CI 1.32 to 2.24; $\mathrm{p}<0.05)$ and first-degree relatives with high CAC (OR 1.49, $95 \%$ CI 1.09 to $2.04 ; \mathrm{p}<0.05$ ) compared with controls (table 3 ). Furthermore, we found that PWV was positively associated with patients (OR 1.41, 95\% CI 1.11 to 1.79 ; $\mathrm{p}<0.05$ ) compared with first-degree relatives with normal CAC. These associations remained after adjustment for confounders.

\section{DISCUSSION}

In this prospective case-control study, we show that PWV is increased in both patients with premature $\mathrm{CAD}$ as well as in firstdegree relatives without overt CVD. These data imply that apparently healthy asymptomatic first-degree relatives of patients with premature $\mathrm{CAD}$ display features of stiffened arteries at a relatively young age, suggesting established vascular damage.

The increased PWV in first-degree relatives of patients with premature $\mathrm{CAD}$ is in agreement with previous studies showing signs of subclinical atherosclerosis in individuals with a positive family history of CAD. Earlier, it was found that in 1662 subjects a family history of premature $\mathrm{CAD}$ was independently correlated with carotid intima media thickness, whereas a family history of late-onset CAD was not. ${ }^{19}$ Other studies confirm these findings with regard to intima media thickness; not only in adults, ${ }^{20}$ but even in children with a positive family history. ${ }^{21}$ Previous studies have also shown that CAC is associated with a positive family history of $\mathrm{CAD} .^{22}$

With respect to the patients with premature $\mathrm{CAD}$, it is known that various conditions such as hypertension ${ }^{23}$ and endstage renal disease ${ }^{24}$ and diabetes ${ }^{25}$ are associated with the development of arterial stiffness. The first-degree relatives had a higher prevalence of classic risk factors compared with controls, which are also associated with an increased PWV. However, the Framingham risk score was comparable and after correction for classic risk factors in relatives and patients, PWV remains higher, which could indicate a specific hereditary component.

In the past few years, the genetic component of CAD has received much attention. By means of genome-wide association studies, several candidate genes were found to be associated with $\mathrm{CAD}^{26}$ The causative pathway for these genes is mostly unknown, but accelerated arterial stiffening could be a possible mechanism. In line with this, the impact of heritable factors on PWV has recently been confirmed, ${ }^{27}$ but it is unknown whether PWV plays a role in familial CAD. More research is needed in this field.

The striking elevation of PWV-up to $15 \mathrm{~m} / \mathrm{s}$-in these apparently 'healthy' individuals with a genetic predisposition towards premature $\mathrm{CAD}$ could imply a causal role in the development of premature CAD. Therefore, PWV measurements might be a practical tool for risk assessment, because the classic risk factors fail to operate in this particular high-risk subset of individuals. ${ }^{6}$ Indeed, a previous study found that a high PWV in subjects with a low SCORE risk $(<5 \%)$, was a strong predictor of cardiovascular events, ${ }^{9}$ suggesting a better risk prediction by PWV in these individuals. Furthermore, another study showed the same disagreement between the Framingham risk score and PWV, in which the highest predictive value of PWV for CAD was found in subjects with a low Framingham risk score. ${ }^{28}$ This implies that PWV is particularly useful in younger individuals, as age is the major contributor in these models. Whether PWV is indeed a good predictor of premature $\mathrm{CAD}$ in these relatives needs to be confirmed in prospective trials.

The limitations of our study merit some consideration. A major limitation of this study is that we do not have follow-up data to establish the true predicted value of PWV in first-degree relatives of patients with premature $\mathrm{CAD}$. A second limitation is that the Arteriograph is a relatively new device for the assessment of PWV. Despite the fact that it has been validated in some studies, to date no prospective outcome studies have been performed with the Arteriograph. A third limitation is that, assuming a hereditary component in the families, the firstdegree relatives probably consisted of individuals who have and who have not inherited the genetic defect. This could be the reason for the large distribution in this group. Ideally, there would be a method to test for the unknown genetic defect. We have tried to substitute this by performing a CAC score. Prospective follow-up studies show that CAC predicts cardiovascular events, independent of other risk factors. ${ }^{29}{ }^{30}$ Also, the elevated CAC score is highly abnormal in these young individuals. Taking into account the logistic regression, the association with PWV increases in the first-degree relatives with abnormal CAC, whereas it decreases in the group with normal CAC, compared with analysing the first-degree relatives as one group. However, we do not know whether the increased familial risk in first-degree relatives co-segregates with an elevated CAC score.

In conclusion, we found that first-degree relatives without overt CVD of patients with premature CAD had higher PWV compared with unrelated controls, independent of other risk factors. Interestingly, a high PWV was related to first-degree relatives with high $\mathrm{CAC}$ scores, whereas it was not in firstdegree relatives with a normal CAC score. This holds promise for the future, in which arterial stiffness could play a role in risk prediction within families with premature CAD. However, to be able to evaluate the prognostic value of PWV, prospective studies in families with premature $\mathrm{CAD}$ are needed.

Competing interests None.

Patient consent Obtained. 
Ethics approval This study was approved by the Institutional Review Board of the Academic Medical Centre, Amsterdam.

Provenance and peer review Not commissioned; externally peer reviewed.

\section{REFERENCES}

1. Andresdottir MB, Sigurdsson G, Sigvaldason H, et al. Fifteen percent of myocardial infarctions and coronary revascularizations explained by family history unrelated to conventional risk factors. The Reykjavik Cohort Study. Eur Heart J 2002;23:1655-63.

2. Lloyd-Jones DM, Nam BH, D'Agostino RB Sr, et al. Parental cardiovascular disease as a risk factor for cardiovascular disease in middle-aged adults: a prospective study of parents and offspring. JAMA 2004;291:2204-11.

3. Sivapalaratnam S, Boekholdt SM, Trip MD, et al. Family history of premature coronary heart disease and risk prediction in the EPIC-Norfolk prospective population study. Heart 2010;96:1985-9.

4. Nasir K, Michos ED, Rumberger JA, et al. Coronary artery calcification and family history of premature coronary heart disease: sibling history is more strongly associated than parental history. Circulation 2004;110:2150-6.

5. Rissanen AM. Familial occurrence of coronary heart disease: effect of age at diagnosis. Am J Cardiol 1979;44:60-6.

6. Sailam V, Karalis DG, Agarwal A, et al. Prevalence of emerging cardiovascular risk factors in younger individuals with a family history of premature coronary heart disease and low Framingham risk score. Clin Cardiol 2008;31:542-5.

7. Laurent S, Cockcroft J, Van Bortel L, et al. Expert consensus document on arterial stiffness: methodological issues and clinical applications. Eur Heart $J$ 2006;27:2588-605.

8. Hansen TW, Li Y, Staessen JA, et al. Independent prognostic value of the ambulatory arterial stiffness index and aortic pulse wave velocity in a general population. J Hum Hypertens 2008;22:214-16.

9. Sehestedt T, Jeppesen J, Hansen TW, et al. Risk prediction is improved by adding markers of subclinical organ damage to SCORE. Eur Heart $J$ 2010;31:883-91.

10. Hauser ER, Mooser V, Crossman DC, et al. Design of the Genetics of Early Onset Cardiovascular Disease (GENECARD) study. Am Heart J 2003;145:602-13.

11. National Cholesterol Education Program (NCEP) Expert Panel on Detection, Evaluation, and Treatment of High Blood Cholesterol in Adults (Adult Treatment Panel III). Third Report of the National Cholesterol Education Program (NCEP) Expert Panel on Detection, Evaluation, and Treatment of high blood cholesterol in adults (Adult Treatment Panel III) final report. Circulation 2002;106:3143-421

12. Jatoi NA, Mahmud A, Bennett $K$, et al. Assessment of arterial stiffness in hypertension: comparison of oscillometric (Arteriograph), piezoelectronic (Complior) and tonometric (SphygmoCor) techniques. J Hypertens 2009;27:2186-91.

13. Horvath IG, Németh A, Lenkey Z, et al. Invasive validation of a new oscillometric device (Arteriograph) for measuring augmentation index, central blood pressure and aortic pulse wave velocity. J Hypertens 2010;28:2068-75.
14. Trachet B, Reymond P, Kips J, et al. Numerical validation of a new method to assess aortic pulse wave velocity from a single recording of a brachial artery waveform with an occluding cuff. Ann Biomed Eng 2010;38:876-88.

15. Mancia G, De Backer G, Dominiczak A, et al. 2007 ESH-ESC Practice Guidelines fo the Management of arterial hypertension: ESH-ESC Task Force on the Management of arterial hypertension. J Hypertens 2007:25:1751-62.

16. Agatston AS, Janowitz WR, Hildner FJ, et al. Quantification of coronary artery calcium using ultrafast computed tomography. J Am Coll Cardiol 1990;15:827-32

17. Arad Y, Spadaro LA, Roth M et al. Treatment of asymptomatic adults with elevated coronary calcium scores with atorvastatin, vitamin $\mathrm{C}$, and vitamin $\mathrm{E}$ : the St Francis Heart Study randomized clinical trial. J Am Coll Cardiol 2005;46:166-72.

18. Cecelja M, Chowienczyk P. Dissociation of aortic pulse wave velocity with risk factors for cardiovascular disease other than hypertension: a systematic review. Hypertension 2009;54:1328-36.

19. Wang TJ, Nam BH, D'Agostino RB, et al. Carotid intima-media thickness is associated with premature parental coronary heart disease: the Framingham Heart Study. Circulation 2003:108:572-6.

20. Anderwald C, Stadler M, Golay A, et al. Impact of family history on relations between insulin resistance, LDL cholesterol and carotid IMT in healthy adults. Heart 2010;96:1191-200.

21. Barra S, Gaeta G, Cuomo S, et al. Early increase of carotid intima-media thickness in children with parental history of premature myocardial infarction. Heart 2009;95:642-5.

22. Nasir K, Budoff MJ, Wong ND, et al. Family history of premature coronary heart disease and coronary artery calcification: Multi-Ethnic Study of Atherosclerosis (MESA). Circulation 2007;116:619-26.

23. Laurent $\mathbf{S}$, Boutouyrie P. Recent advances in arterial stiffness and wave reflection in human hypertension. Hypertension 2007:49:1202-6.

24. Blacher J, Safar ME, Guerin AP, et al. Aortic pulse wave velocity index and mortality in end-stage renal disease. Kidney Int 2003;63:1852-60.

25. Cruickshank K, Riste L, Anderson SG, et al. Aortic pulse-wave velocity and its relationship to mortality in diabetes and glucose intolerance: an integrated index of vascular function? Circulation 2002;106:2085-90.

26. Schunkert H, König IR, Kathiresan S, et al. Large-scale association analysis identifies 13 new susceptibility loci for coronary artery disease. Nat Genet 2011:43:333-8.

27. Mitchell GF, DeStefano AL, Larson MG, et al. Heritability and a genome-wide linkage scan for arterial stiffness, wave reflection, and mean arterial pressure: the Framingham Heart Study. Circulation 2005;112:194-9.

28. Boutouyrie $\mathbf{P}$, Tropeano Al, Asmar $\mathrm{R}$, et al. Aortic stiffness is an independent predictor of primary coronary events in hypertensive patients: a longitudinal study. Hypertension 2002;39:10-15.

29. Budoff MJ, Shaw LJ, Liu ST, et al. Long-term prognosis associated with coronary calcification: observations from a registry of 25,253 patients. J Am Coll Cardiol 2007:49:1860-70.

30. Shaw LJ, Raggi P, Schisterman E, et al. Prognostic value of cardiac risk factors and coronary artery calcium screening for all-cause mortality. Radiology 2003;228:826-33. 